\title{
Surgical pearls: combined retinal detachment and choroidal detachment - Can you insert an infusion cannula?
}

\author{
Rashed Mustafa Nazzal (i), Katarzyna Nowomiejska (ㄷ, Robert Rejdak (1) \\ Department of General Ophthalmology, Medical University of Lublin, Lublin, Poland
}

\begin{abstract}
Combined retinal detachment and choroidal detachment is an uncommon diagnosis that a surgeon will occasionally face. Although the experienced vitreoretinal surgeon can manage nearly all obstacles during the surgery, an inexperienced vitreoretinal surgeon will face a few difficulties during certain steps. In this article, we describe a case report and highlight some of these obstacles and suggest some options to overcome them.
\end{abstract}

KEY WORDS: choroidal detachment; retinal detachment; anterior chamber maintainer; infusion cannula

Ophthalmol J 2019; Vol. 4, 60-63

\section{INTRODUCTION}

The incidence of combined retinal detachment and choroidal detachment is infrequent, affecting only $2-4.5 \%$ of all rhegmatogenous retinal detachment cases [1-3], with a higher incidence among the Chinese population (up to 18.1\%) [4].

The risk increases in patients with high myopia, aphakia, pseudophakia, and advanced age, who usually present with low intraocular pressure and multiple retinal breaks [2].

This special type of retinal detachment is known to have a higher rate of failure if managed with buckle surgery, for many reasons such as poor visualisation, difficult identification of causative breaks, difficult application of retinopexy, and the development of proliferative vitreoretinopathy; hence, management by vitrectomy is usually preferred, but even then, it is still considered to be a preoperative risk factor for failure $[3,5]$.

\section{CASE REPORT}

A 61-year-old male patient, pseudophakic, presented with painless loss of vision in the right eye of a few weeks' duration. On examination his best corrected visual acuity was hand motion, intraocular pressure was $5 \mathrm{mmHg}$, anterior segment examination showed a poorly dilating pupil, intraocular lens in place, and poor visualisation to the fundus because of the small pupil and vitreous haemorrhage. B-scan ultrasonography was done (Fig. 1) and revealed vitreous haemorrhage, retinal detachment with visible retinal break, and choroidal detachment. The choroidal detachment was explained on the basis of retinal detachment-induced hypotony. During the operation, 25G trocar sets were used. The first trocar (the infusion) was inserted in the inferotemporal quadrant with difficulty because of the existent hypotony; the tip of the trocar was ascertained to be in the vitreous cavity using an 


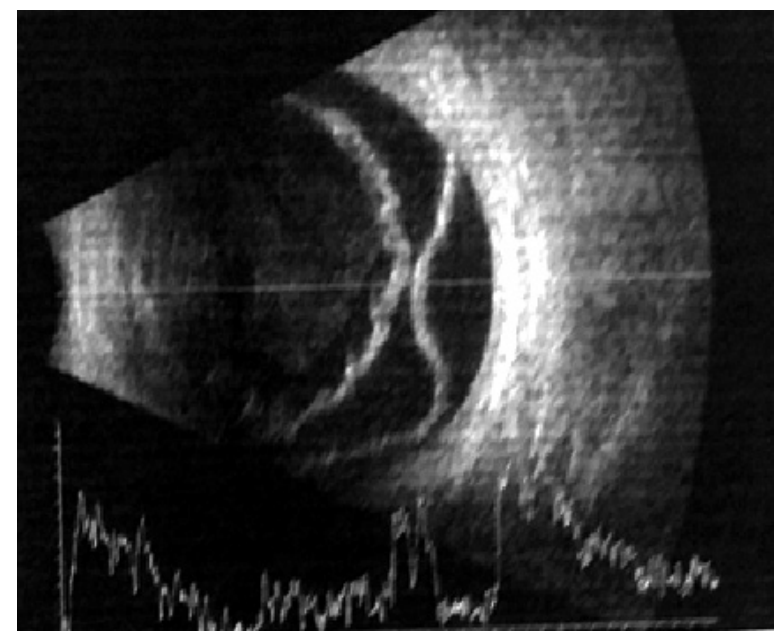

FIGURE 1. B-scan ultrasonography showing vitreous haemorrhage, retinal detachment, and choroidal detachment in addition to the classical M-sign spike of choroidal detachment in the incorporated A-scan

external light beam. The infusion line was opened, the intraocular pressure increased, and the second and third trocars were inserted. During early core vitrectomy the infusion line was noted to disappear from the view, so the infusion was stopped immediately and a few seconds were taken to assess the situation. It was assumed that the infusion cannula was retracted in the suprachoroidal space, given the already present choroidal detachment, so a trial was done to insert it again through the same place without success. Two other sites of insertion were tried, but both failed. A decision was made to insert an anterior chamber maintainer to pressurise the eye. It was inserted successfully, and vitrectomy was resumed. But given that we would need to perform a fluid-air exchange later on in the surgery, continuing using the anterior chamber maintainer was not a good strategy. After doing central and peripheral vitrectomy, the retina was flat and the choroids were noted to slightly decrease in size, so another attempt to insert the infusion cannula was made at the 12 o'clock position (Fig. 2) in the place of least choroidal detachment as seen directly by endoillumination. It was successful. The anterior chamber maintainer was removed and the vitrectomy was completed with silicon oil tamponading the eye at the end of the surgery. During the surgery, the patient was found to have five retinal holes, two of them just posterior to the equator. Stage B proliferative vitreoretinopathy was also observed.

\section{DISCUSSION}

Although the exact mechanism of combined choroidal detachment and rhegmatogenous retinal detachment is not clear yet, most investigators believe that hypotony induced by retinal detachment is the first stage of choroidal detachment. Liquefied vitreous enters the subretinal space through sensory retinal defects and stimulates choroidal abnormalities, including dilatation and hyperpermeability of choroidal vessels. The gradually increased exudation of fluid into the suprachoroidal space results in choroidal detachment. In addition, oedema of the ciliary body further reduces the generation of aqueous humour and causes prominent hypotony, deteriorating the dilatation and hyperpermeability of choroidal vessels and leading to progression of

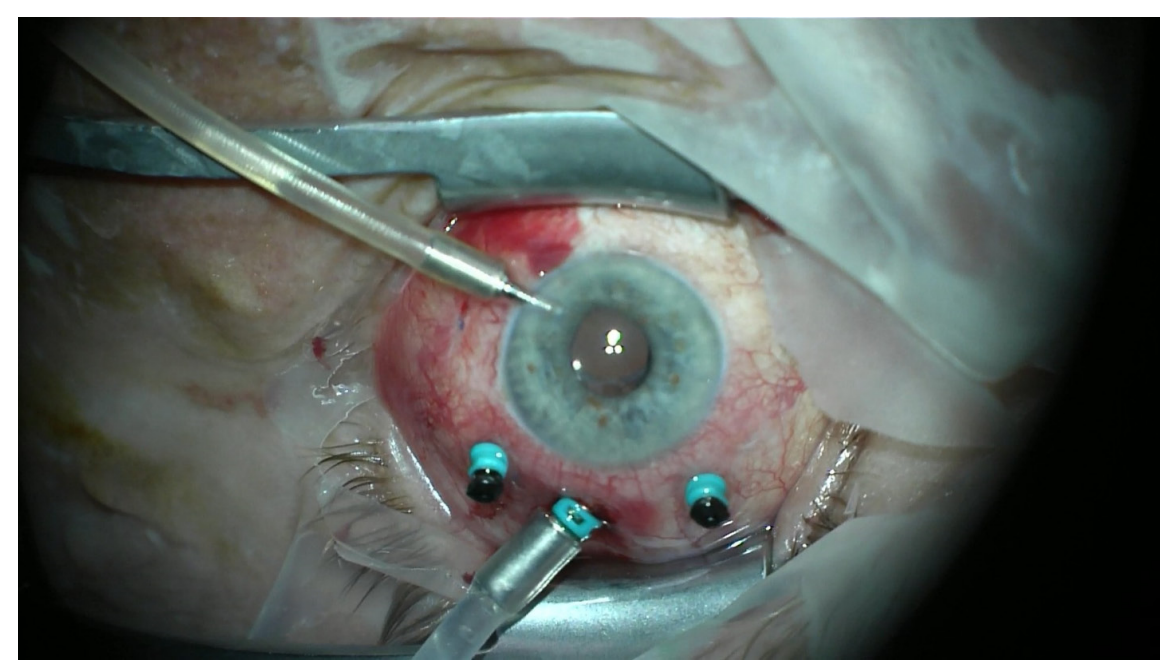

FIGURE 2. Photo demonstrating the position of the infusion at the 12 o'clock position. Notice the anterior chamber maintainer 
choroidal detachment. Therefore, a positive feedback loop is established $[6,7]$.

With the advent of 23- and 25-gauge pars plana vitrectomy, it has been postulated that the risk of intraoperative choroidal detachment may be increased compared with that of 20 -gauge vitrectomy because the infusion port is sutured in place in 20 -gauge vitrectomy, while 23 - and 25 -gauge vitrectomy systems use self-fixating, un-sutured cannulas, which may be more likely to retract and subsequently infuse into the suprachoroidal space [8].

Various types of intraoperative choroid detachments have been described, such as serous, haemorrhagic, sulphur hexafluoride (SF6) gas, air, and silicon oil [8-10].

Sometimes, placing cannulas at the standard positions is not safe; for example, when there is difficulty with direct visualisation or difficulty with anatomical barriers such as trauma, endophthalmitis, dense vitreous haemorrhage, and appositional choroidal detachment [8].

For an experienced retinal surgeon, dealing with difficulties during the surgery might be an easy job, but for the beginner it might not be an easy mission. The first thing the surgeon should do, is to rule out any underlying cause for the choroidal detachment, most importantly tumours and the possibility that this choroidal detachment is due to exudative retinal detachment rather than a rhegmatogenous retinal detachment, which can be done by fundus examination, if the view is good, or by an ultrasonography in the case of a hazy view to the fundus. A B-scan will show the absence of an underlying mass and will demonstrate characteristic features of choroidal detachment, and it can show the retinal break in some cases. Once the diagnosis is established, the surgeon should make the decision of whether to pre-treat with steroids or not [5]. Then the surgeon should be prepared for the initial step in the surgery, which is the insertion of the infusion cannula. Before the infusion line is opened, the tip of the cannula must be checked. A brief peek is not enough. The choroid and ciliary epithelium may be depigmented, and a quick peek will not allow the surgeon to detect if something is covering the tip of the cannula. And even an initially properly placed infusion cannula may be pulled out intraoperative. It is easy to detect if the line is completely out, but it is more difficult if only partially so. The position of the infusion cannula's external position should be periodically checked during surgery, especially during scleral indentation [11].
If, during the initial cannula insertion, the tip of the cannula cannot be advanced all the way through the tissue, there are several options. First, push a needle through the cannula itself; this will create a small opening in the choroid, which may slide back. If this does not work (it may not because the choroid is elastic, and the opening may immediately close), introduce the cannula in another place, insert a needle through that one, indent the sclera by pushing the first cannula in, and cut open the tissue around it so that there is a clear view of the tip. Bleeding is extremely rare from this. Do not use diathermy because it may shrink the tissue [11].

If fluid does get under the choroid, close the infusion immediately, pull out the infusion line, and put it through another cannula and check its position before opening it. Or, in the case of a partially disinserted cannula, leave it in position and use it to drain the suprachoroidal fluid [12]. If no fluid flows or not all of fluid drains, pull out the cannula and enlarge the sclerotomy. Once the fluid has been drained, suture this sclerotomy and reinsert the infusion cannula at a different location.

Other options to use if you cannot get a view for the tip of the cannula include: use of a long 6-mm cannula specifically designed for this purpose, but one should be careful not to hit the lens should the patient be phakic. Alternatively, the $6-\mathrm{mm}$ cannula from the 20G vitrectomy set can be used (but the cannula must be removed and the sclerotomy enlarged). If all of these methods fail and it is impossible to insert a cannula in the vitreous cavity, then an anterior chamber maintainer may be used. The classical type has a few drawbacks: spontaneous extrusion and forced introduction into the eye from variability in the size of the corneal paracentesis incision and the possibility of damaging the endothelium. Hence, you can use trocars that are designed for the posterior chamber, $23 \mathrm{G}$ or $25 \mathrm{G}$, by using a trans-conjunctival bi-planar wound of appropriate size that is self-sealing and overcomes the shortcomings of an anterior chamber maintainer. Constant infusion inflow through the trocar anterior chamber maintainer is used to maintain positive intraocular pressure through a self-sealing sclerotomy incision at the limbus [13]. Another option is to use a new, specially designed $25 \mathrm{G}$ anterior chamber maintainer [14].

\section{CONCLUSION}

Few steps in vitreoretinal surgery look easy, but sometimes surgeons are faced with unexpected situ- 
ations in which they have to manage immediately with a good step-wise approach. Knowing the options to manage every step of the surgery will increase one's confidence and readiness to deal with these unexpected events.

\section{ACKNOWLEDGEMENTS}

None.

\section{STATEMENT OF COMPETING INTERESTS}

None.

\section{REFERENCES}

1. Gottlieb F. Combined choroidal and retinal detachment. Arch Ophthalmol. 1972; 88(5): 481-486, doi: 10.1001/archopht.1972.01000030483003, indexed in Pubmed: 4634785.

2. Seelenfreund MH, Kraushar MF, Schepens CL, et al. Choroidal detachment associated with primary retinal detachment. Arch Ophthalmol. 1974; 91(4): 254-258, doi: 10.1001/archopht.1974.03900060264003, indexed in Pubmed: 4621149.

3. Sharma T, Challa JK, Ravishankar KV, et al. Scleral buckling for retinal detachment. Predictors for anatomic failure. Retina. 1994; 14(4): 338-343, doi: 10.1097/00006982-199414040-00008, indexed in Pubmed: 7817027.

4. Zhu J, XuX, Zhang Xi. [Surgical therapeutic results of rhegmatogenous retinal detachment associated with choroidal detachment]. Zhonghua Yan Ke Za Zhi. 2002; 38(3): 135-139, indexed in Pubmed: 11955315.

5. Sharma T, Gopal L, Reddy RK, et al. Primary vitrectomy for combined rhegmatogenous retinal detachment and choroidal detachment with or without oral corticosteroids: a pilot study. Retina. 2005; 25(2):
152-157, doi: 10.1097/00006982-200502000-00006, indexed in Pubmed: 15689804.

6. Langham ME, Regan CD. Circulatory changes associated with onset of primary retinal detachment. Arch Ophthalmol. 1969; 81(6): 820-829, doi: 10.1001/archopht.1969.00990010822013, indexed in Pubmed: 5783753.

7. Yu Y, An M, Mo B, et al. Risk factors for choroidal detachment following rhegmatogenous retinal detachment in a chinese population. BMC Ophthalmol. 2016; 16: 140, doi: 10.1186/s12886-016-0319-9, indexed in Pubmed: 27507568.

8. Tarantola RM, Folk JC, Shah SS, et al. Intraoperative choroidal detachment during 23-gauge vitrectomy. Retina. 2011; 31(5): 893-901, doi: 10.1097/IAE.0b013e3181f4429b, indexed in Pubmed: 21273944.

9. Lin CJ, Peng KL. Intraoperative severe suprachoroidal air as a complication of 23-gauge vitrectomy combined with air-fluid exchange. Int Med Case Rep J. 2018; 11: 173-176, doi: 10.2147/IMCRJ.S163085, indexed in Pubmed: 30104906.

10. Uji A. Suprachoroidal gas injection as a complication of pars plana vitrectomy confirmed by computed tomography. Clin Ophthalmol. 2012; 6: 533-536, doi: 10.2147/0PTH.S29185, indexed in Pubmed: 22536048.

11. Kuhn F. Vitreoretinal Surgery: Strategies and Tactics. Springer 2016.

12. Witkin AJ, Fineman $M, H_{0} A C$, et al. A novel method of draining intraoperative choroidal detachments during 23-gauge pars plana vitrectomy. Arch Ophthalmol. 2012; 130(8): 1048-1050, doi: 10.1001/ archophthalmol.2012.1472, indexed in Pubmed: 22893075.

13. Agarwal A, Narang P, Kumar DA, et al. Trocar anterior chamber maintainer: Improvised infusion technique. J Cataract Refract Surg. 2016; 42(2): 185-189, doi: 10.1016/j.jcrs.2016.01.015, indexed in Pubmed: 27026440.

14. Narang P, Agarwal A, Kumar DA, et al. Twenty-five-gauge trocar anterior chamber maintainer: New device for infusion. $\mathrm{J}$ Cataract Refract Surg. 2018; 44(7): 797-801, doi: 10.1016/j.jcrs.2018.03.021, indexed in Pubmed: 29807679. 